\title{
Cardio metabolic Risk Factors in Obese Subjects
}

\author{
Odum E.P. ${ }^{1}$, Asekomeh E.G. ${ }^{2}$, Ntuen N.A. ${ }^{3}$, Young E.E. ${ }^{4}$ \\ $M B B S, F M C P a t h ; M B B S, M S c, M P H, F W A C P ; M B B S, F M C P a t h ; M B B S, F W A C P$ \\ ${ }^{1,3}$ Department of Chemical Pathology, University of Port Harcourt Teaching Hospital, Port Harcourt, Rivers \\ State. \\ ${ }^{2}$ Chevron Clinic, Port Harcourt, Rivers State. \\ ${ }^{4}$ Department of Medicine, University of Nigeria, Enugu Campus, Enugu State.
}

\begin{abstract}
Numerous studies have demonstrated the association between obesity and increased risk and prevalence of cardiometabolic conditions, particularly type 2 diabetes and cardiovascular disease (CVD).

This study was conducted to determine if obese subjects have a higher risk and an increased prevalence of type 2 diabetes and other cardiovascular risk factors compared to controls.

Body mass index, waist circumference, blood pressure, fasting glucose and lipids were determined in 150 obese subjects and 157 healthy controls. The prevalence and risk of hypertension, dyslipidaemia, impaired fasting glucose, type 2 diabetes and metabolic syndrome were evaluated.

Obese subjects had a higher prevalence of hypertension, dyslipidaemia, impaired fasting glucose, type 2diabetes and metabolic syndrome and a 2-to 8-fold higher risk of having cardiometabolic comorbidities than controls.

The prevalence and risk of cardiometabolic risk factors were higher in obese subjects compared to controls. Lifestyle changes can bring about weight loss and reduce the risk and prevalence of cardiometabolic complications in obese individuals.
\end{abstract}

Keywords: Cardiometabolic risk factors, obese subjects, metabolic syndrome, type 2 diabetes, cardiovascular disease.

\section{Introduction}

Obesity is a chronic medical condition and a major public health challenge globally [1,2]. The prevalence of obesity has been on an exponential increase, not only in developed countries but also in developing nations, particularly those that have adopted a western lifestyle [1,2]. Far from being a cosmetic problem, it augments the risk of many important diseases [1,2]. Central (abdominal) obesity, in particular, is a potent risk factor for the development of type 2 diabetes mellitus, hypertension, metabolic syndrome and cardiovascular disease (CVD) [1-3].

Obesity is multifactorial in origin, caused by an interplay of genetic susceptibility, excessive food intake and a sedentary lifestyle [1-3]. Adipose tissue, particularly white adipose tissue, is a recognized endocrine organ that releases adipokines, including inflammatory cytokines, into the blood stream [4,5]. Also, as a result of the increased fat mass in obese individuals, there is increased release of free fatty acids (FFA) [5]. All these factors activate inflammatory pathways and impair insulin signalling [5,6]. Obesity is thus associated with insulin resistance, dysregulated glucose and lipid metabolism, low-grade inflammation, excessive oxidative stress, prothrombotic and proatherogenic state, all of which promote the development of cardiometabolic conditions [5].

Obesity has been reported to impart a 3- to 6-fold increase to the risk of developing diabetes mellitus and to account for about $60 \%$ of all cases of diabetes ${ }^{6}$. Large scale studies have shown that the risk of diabetes and cardiovascular disease increases progressively with body mass index (BMI) [2,6,7]. In the Nurses' Health Study, compared with women with a lower BMI, a 28 -fold higher risk of developing diabetes was observed in those with a BMI of $30 \mathrm{Kg} / \mathrm{m}^{2}$. This increased to 93 -fold for women with BMI $>35 \mathrm{Kg} / \mathrm{m}^{2}$. [2]. Obesity has also been demonstrated to reduce life expectancy and is one of the leading preventable causes of death worldwide. A BMI $>32 \mathrm{Kg} / \mathrm{m}^{2}$ has been shown to double mortality rate; a BMI of $30-35 \mathrm{Kg} / \mathrm{m}^{2}$ has been estimated to reduce life expectancy by $2-4$ years and a BMI $>40 \mathrm{Kg} / \mathrm{m}^{2}$ by 10 years [7].

The prevalence of metabolic syndrome and other cardiometabolic risk factors has also been observed to increase with increasing BMI, as well as with increasing waist circumference, in all weight categories (normal weight, overweight and obese individuals) $[8,9]$.

Modest weight loss of $5 \%-10 \%$ has been associated with increased insulin sensitivity, improved glucose and lipid metabolism, decreased blood pressure, decreased burden of multiple risk factors and increased life expectancy [4,6,7]. Lifestyle modification may lead to a $60 \%$ reduction in risk of diabetes and a $37 \%$ reduction in metabolic syndrome prevalence [4]. 
The aim of this study is to determine the prevalence and risk of cardiometabolic conditions in obese subjects compared to controls.

\section{Subjects And Methods}

The study population was made up of a total of 150 obese subjects selected from patients attending the General Outpatient and Metabolic Clinics of the University of Port Harcourt Teaching Hospital (UPTH) and 157 controls which included apparently healthy volunteers consecutively enrolled from hospital staff, medical students and relatives of patients attending the metabolic, general and medical out-patient clinics of UPTH. Ethical approval was obtained from the hospital's Research Ethical Committee.

Informed consent was given by participants and necessary clinical information was obtained from them with the use of a standardized pretested questionnaire. Participants that were below 18 years, pregnant, acutely or chronically ill, had a history of diabetes mellitus or cardiovascular disease, heavy smokers and alcoholics were excluded. After a brief general examination, the blood pressure (BP) of each participant was measured using a standard mercury sphygmomanometer, waist circumference (WC) was measured just above the iliac crest and BMI was estimated from the height and weight.

Venous blood samples were collected after an overnight fast. Fasting plasma glucose (FPG) concentration was determined using the glucose oxidase method and plasma triglyceride, total cholesterol and high density lipoprotein (HDL)-cholesterol were estimated by enzymatic methods. Low density lipoprotein (LDL)-cholesterol was calculated from the Friedewald formula [10].

The following definitions were used in this study:

Obesity: Defined as BMI $\geq 30 \mathrm{Kg} / \mathrm{m}^{2}$ and classified as class I obesity (BMI of 30.0-34.9), class II obesity (BMI of 35.0-39.9) and class III obesity (BMI $\geq 40)$ [3].

Hypertension: Defined as systolic blood pressure equal to or above $140 \mathrm{mmHg}$ and diastolic blood pressure equal to or above $90 \mathrm{mmHg}$ [3].

Metabolic Syndrome: Based on the NCEP-ATP III (2005) definition, any three or more of the following criteria: hyperglycaemia (FPG $\geq 5.6 \mathrm{mmol} / \mathrm{L}$ ), abdominal obesity $(\mathrm{WC}>102 \mathrm{~cm}$ in men and $>88 \mathrm{~cm}$ in women), $\mathrm{BP} \geq 130 / 85 \mathrm{mmHg}$, triglyceride (TG) $\geq 1.7 \mathrm{mmol} / \mathrm{L}, \mathrm{HDL}<1.0 \mathrm{mmol} / \mathrm{L}$ (men) and $<1.3 \mathrm{mmol} / \mathrm{L}$ (women) [11].

Impaired fasting glucose: FPG: $6.1-6.9 \mathrm{mmol} / \mathrm{L}$ [11].

Type 2 diabetes (screen-detected): Based on a FPG $\geq 7.0 \mathrm{mmol} / \mathrm{L} \mathrm{[3]}$.

Dyslipidaemia: Defined as any one or more of hypercholesterolaemia (plasma total cholesterol $\geq 5.2 \mathrm{mmol} / \mathrm{L}$ ), hypertriglyceridaemia (plasma triglyceride $\geq 1.7 \mathrm{mmol} / \mathrm{L}$ ), high LDL (plasma LDL-cholesterol $\geq 2.6 \mathrm{mmol} / \mathrm{L}$ ) and low HDL (HDL-cholesterol $<1.0 \mathrm{mmol} / \mathrm{L}$ ) [3].

\section{Statistical Analysis}

Statistical analysis was done using the Statistical Package for Social Sciences (SPSS) software version 17.0 (SPSS Inc., Chicago, Illinois, USA). Means of continuous variables were compared using the unpaired student-t test and expressed as mean \pm standard deviation (SD). Pearson correlation coefficient was used to assess the relationship between BMI and waist circumference. Frequencies and percentages were obtained for categorical variables. Pearson's chi square or Fishers exact test as appropriate was used to test for differences between categorical variables. Binary logistic regression analysis was used to identify factors that were significantly associated with obesity using the BMI and findings were presented as odds ratio (OR), its $95 \%$ confidence interval and corresponding P-values. A level of $\mathrm{p} \leq 0.05$ was considered statistical significant in all analyses.

\section{Results}

There were 150 obese subjects with a mean age of $42.6 \pm 11.6$ years and 157 control individuals with a mean age of $34.2 \pm 13.1$ years. There was a significant difference between the mean ages of obese subjects and controls $(p<0.001)$. Majority of the obese subjects $(57.3 \%)$ were in the age range of $30-49$ years. $91(60.7 \%)$ of the obese subjects were women and $59(39.3 \%)$ were men.

Most of the obese subjects $(60 \%)$ had class I obesity while $29.1 \%$ and $10.9 \%$ had classes II and III obesity respectively. Among the controls, $66.2 \%$ were normal weight and $33.8 \%$ were overweight. None of them was obese.

The mean BMI, waist circumference, blood pressure, fasting plasma glucose and triglyceride of obese subjects were significantly higher than that of controls $(\mathrm{p}<0.001$ for all). The mean LDL was also significantly higher in obese subjects $(\mathrm{p}=0.03)$ but total cholesterol and HDL were not significantly different between the two groups (Table 1). Pearson correlation statistics showed a strong correlation between BMI and waist circumference $(r=0.844, \mathrm{p}<0.001)$ in the total population of obese subjects and controls. 
Obese subjects had a higher prevalence of abdominal obesity (82.7\%), hypertension (57.3\%), dyslipidaemia, metabolic syndrome (30.7\%), impaired fasting glucose $(9.3 \%)$ and type 2 diabetes $(6.7 \%)$ compared to the controls (Table 2).

BMI was significantly associated with older age ( $\geq 45$ years), hypertension, abdominal obesity, hyperglycaemia, impaired fasting glucose, hypertriglyceridaemia, low HDL, total cholesterol and metabolic syndrome but not with type 2 diabetes and LDL (Tables 2 and 3). Obese subjects had approximately 31-fold higher risk of having abdominal obesity and 2- to 8-fold increased risk of developing the other risk factors compared to controls (Table 3).

Prevalence of risk factors increased progressively with increasing BMI across different BMI categories in obese subjects and controls. The trend of increasing prevalence of risk factors with increase in BMI in the control subjects was significant only for abdominal obesity. In the obese subjects however, there was a significant trend of increasing prevalence of abdominal obesity, hypertension and metabolic syndrome with increasing BMI. The peak prevalence of abdominal obesity, hypertension, hypertriglyceridaemia, low HDL, impaired fasting glucose and metabolic syndrome occurred in the BMI class greater than $40 \mathrm{Kg} / \mathrm{m}^{2}$ while that of type 2 diabetes, hypercholesterolaemia and high LDL occurred in the BMI class of $35-39.9 \mathrm{Kg} / \mathrm{m}^{2}$ (Table 4).

\section{Discussion}

It has been well established that obesity promotes insulin resistance and the development of metabolic syndrome, type 2 diabetes and CVD [2,12]. Several studies have demonstrated that the development of obesityrelated comorbidities is not simply a direct consequence of increased fat mass but associated with type of fat distribution, duration of obesity, presence of insulin resistance and clustering of risk factors [4,6]. Visceral (abdominal) adiposity, rather than peripheral adiposity, is primarily associated with the development of insulin resistance and related comorbidities [4]. Health consequences of obesity tend to worsen with increasing duration of obesity and multiplicity of risk factors [6,7].

This study revealed that compared to controls obese subjects had a higher prevalence of hypertension, abdominal obesity, dyslipidaemia, impaired fasting glucose, metabolic syndrome and type 2 diabetes. They also had approximately 31-fold higher risk of having abdominal obesity and 2- to 8-fold higher risk of having hypertension, non-LDL dyslipidaemia, impaired fasting glucose and metabolic syndrome. The prevalence of cardiometabolic risk factors increased progressively with increasing BMI. Obese subjects with BMI $>40 \mathrm{Kg} / \mathrm{m}^{2}$ had the highest prevalence of abdominal obesity, hypertension, hypertriglyceridaemia, low HDL, impaired fasting glucose and metabolic syndrome while subjects with BMI between 35 and $39.9 \mathrm{Kg} / \mathrm{m}^{2}$ had the highest prevalence of type 2 diabetes, hypercholesterolaemia and high LDL. The increase in the prevalence of hypertension and metabolic syndrome was more prominent across BMI classes in obese subjects while that of abdominal obesity and type 2 diabetes was more prominent across BMI classes in controls (normal weight and overweight).

In a community-based longitudinal study, Meigs and associates noted that among normal-weight, overweight and obese subjects, the incidence for diabetes was $1.6 \%, 4.1 \%$ and $11.6 \%$ respectively, while for CVD it was $6.0 \%, 9.4 \%$ and $11.8 \%$ respectively. In addition, overweight, obesity, metabolic syndrome or insulin resistance increased the risk for incident diabetes and CVD by about 5- to 7-fold and 1.6- to 2-fold respectively relative to those without these conditions. They also demonstrated increased prevalence of risk factors with increasing BMI [9].

Behan and associate found that in healthy non-obese women, of all the groups studied, women in the highest quartile of waist circumference had the highest BMI, the highest prevalence of hypertension, hypertriglyceridaemia and low HDL, and the highest risk for diabetes and CVD [8]. Waist circumference correlated strongly with BMI. We observed a similar correlation in this study.

When compared to controls, obese subjects in this study, however, did not demonstrate an increased risk for type 2 diabetes but they had approximately 4-fold increased risk of impaired fasting glucose, 4-fold increased risk of hyperglycaemia (which includes impaired fasting glucose and diabetes) and 8-fold increased risk of metabolic syndrome. Metabolic syndrome has been associated with a 3 -fold increased risk of type 2 diabetes and a 2-fold increased risk of CVD [13]. The fasting plasma glucose level of obese subjects was also observed to be higher than that of controls. Many adipokines, including TNF- $\alpha$, IL- 6 and complement C3, secreted by the adipose tissue, have been associated with insulin resistance and impaired glucose tolerance [14]. Obesity-induced insulin resistance increases the allosteric load of the pancreas and coupled with the direct deleterious effect of hyperglycaemia on the beta cell (glucotoxicity) and lipotoxicity, may eventually result in failure of the endocrine pancreas and type 2 diabetes [14].

Hypertension was the most prevalent $(57.3 \%)$ cardiometabolic comorbidity observed in obese subjects. This finding is in consonance with that of previous researchers though lower percentages were reported in earlier studies done in Port Harcourt and other parts of Nigeria [3,15]. Obese subjects in this study had about 6fold higher risk of hypertension relative to controls. Obesity has been reported to be the most common risk 
factor for hypertension, accounting for about $40 \%-72 \%$ of cases $[6,7,16]$. A $10-\mathrm{Kg}$ weight gain is associated with an increase of $3.0 \mathrm{mmHg}$ in systolic and $2.3 \mathrm{mmHg}$ diastolic blood pressure, translating to a $12 \%$ increased risk for CVD and a 24\% increased risk for cerebrovascular accident [4]. Obese individuals have been shown to have a 5-fold increased incidence of hypertension compared to normal weight individuals [17]. The development of hypertension in obese individuals is associated with increased peripheral resistance, increased cardiac output and increased blood volume that occur as a compensatory response to the increased metabolic demand of expanded adipocyte mass [4,16]. It is also associated with insulin resistance and concomitant overactivation of the renin-angiotensin-aldosterone system, increased renal sodium retention, increased sympathetic nervous system activity and stimulation of vascular smooth muscle growth $[7,12]$.

The most frequent lipid disorder observed in this study was high LDL-cholesterol, both in the obese subjects $(38.2 \%)$ and in controls $(29.9 \%)$. Traditional cardiovascular risk factors like high LDL are usually associated with impaired endothelial function and premature atherosclerosis [4]. Previous studies have shown that obesity is particularly associated with hypertriglyceridaemia, low HDL and hypercholesterolaemia [7,12]. As a result of increased insulin resistance there is impaired lipoprotein lipase activity, and increased release of free fatty acids from visceral fat cells provides a greater substrate for hepatic triglyceride and LDL synthesis. This is followed by decreased levels of cardioprotective HDL and Apo A [4,12]. In this study, obese subjects had a higher prevalence of dyslipidaemia than controls and low HDL, hypertriglyceridaemia and hypercholesterolaemia were associated with increased risk (approximately 2-fold, 3-fold and 2-fold respectively) in obese subjects.

\section{Conclusion}

Obese subjects in this study had a higher prevalence of hypertension, abdominal obesity, dyslipidaemia, impaired fasting glucose, metabolic syndrome and type 2 diabetes than controls and this increased with increasing BMI. They also had a higher risk of having CVD risk factors.

This study is however not without some limitations. Being a cross-sectional study, incidence of type 2 diabetes and CVD could not be ascertained. Furthermore, 2-hours oral glucose tolerance test (OGTT) glucose level was not evaluated, thus subjects who would have been diagnosed diabetic based on 2-hours plasma glucose $\geq 11.1 \mathrm{mmol} / \mathrm{L}$ as well as subjects with impaired glucose tolerance were not detected. This could have resulted in an underestimation of the total number of individuals with dysglycaemia (impaired fasting glucose, impaired glucose tolerance and/or diabetes).

In Nigeria and other parts of Africa, obesity is still generally seen as an index of wealth and well-being. Increased awareness is required, particularly through the mass media, to educate the populace about the medical consequences of obesity. Lifestyle changes, by way of increased physical activity, regular exercise and consumption of healthy foods can bring about weight loss and thus help in halting the development and progression of obesity-related cardiometabolic complications.

Table 1: Cardiometabolic risk factors of obese subjects and controls

\begin{tabular}{|llll|}
\hline Risk Factor & $\begin{array}{l}\text { Obese subjects }(\mathbf{n}=150) \\
\text { Mean } \pm \text { SD }\end{array}$ & $\begin{array}{l}\text { Controls }(\mathbf{n}=157) \\
\text { Mean } \pm \text { SD }\end{array}$ & P \\
Age & $42.6 \pm 11.6$ & $34.2 \pm 13.1$ & $<0.001^{*}$ \\
Body mass index $\left(\mathrm{Kg} / \mathrm{m}^{2}\right)$ & $34.8 \pm 5.8$ & $23.5 \pm 2.9$ & $<0.001^{*}$ \\
Waist circumference $(\mathrm{cm})$ & $105 \pm 10.4$ & $81.1 \pm 10.0$ & $<0.001^{*}$ \\
Systolic blood pressure $(\mathrm{mmHg})$ & $131.7 \pm 21.6$ & $118.1 \pm 17.0$ & $<0.001^{*}$ \\
Diastolic blood pressure $(\mathrm{mmHg})$ & $87.1 \pm 14.5$ & $76.8 \pm 11.8$ & $<0.001^{*}$ \\
Fasting plasma glucose $(\mathrm{mmol} / \mathrm{L})$ & $5.2 \pm 1.5$ & $4.5 \pm 1.2$ & $<0.001^{*}$ \\
Triglyceride $(\mathrm{mmol} / \mathrm{L})$ & $1.0 \pm 0.4$ & $0.8 \pm 0.4$ & $<0.001^{*}$ \\
High density lipoprotein $(\mathrm{mmol} / \mathrm{L})$ & $1.3 \pm 0.4$ & $1.4 \pm 0.4$ & 0.136 \\
Total cholesterol $(\operatorname{mmol} / \mathrm{L})$ & $4.2 \pm 1.2$ & $4.0 \pm 1.0$ & 0.081 \\
Low density lipoprotein $(\mathrm{mmol} / \mathrm{L})$ & $2.4 \pm 0.9$ & $2.2 \pm 0.8$ & $0.030^{*}$ \\
\hline
\end{tabular}

* Statistically significant $\mathrm{P} \leq 0.05 ; \mathrm{SD}-$ standard deviation

Table 2: Prevalence of cardiometabolic comorbidities of obese subjects and controls

\begin{tabular}{|llll|}
\hline Risk Factor & $\begin{array}{l}\text { Obese Subjects }(\mathbf{n}=\mathbf{1 5 0}) \\
\text { Number }(\%)\end{array}$ & $\begin{array}{l}\text { Controls }(\mathbf{\%}=157) \\
\text { Number }(\%)\end{array}$ & $\mathbf{P}$ \\
Abdominal obesity & $124(82.7)$ & $21(13.4)$ & $<0.001^{*}$ \\
Hypertension & $86(57.3)$ & $30(19.1)$ & $<0.001^{*}$ \\
Hypertriglyceridaemia & $15(10.0)$ & $5(3.2)$ & $0.022^{*}$ \\
Hypercholesterolaemia & $37(24.7)$ & $23(14.6)$ & $0.043^{*}$ \\
High LDL & $57(38.0)$ & $47(29.9)$ & 0.161 \\
Low HDL & $50(33.3)$ & $32(20.4)$ & $0.015^{*}$ \\
Impaired fasting glucose & $14(9.3)$ & $4(2.5)$ & $<0.001^{*}$ \\
Metabolic Syndrome & $46(30.7)$ & $8(5.1)$ & $<0.001^{*}$ \\
Type 2 diabetes & $10(6.7)$ & $6(3.8)$ & 0.347 \\
\hline
\end{tabular}

$\mathrm{LDL}=$ Low density lipoprotein; HDL $=$ High density lipoprotein 
*Statistically significant $\mathrm{P} \leq 0.05$

Table 3: Binary logistic regression of CVD risk factors in obese subjects and controls

\begin{tabular}{|c|c|c|c|c|c|}
\hline Risk Factor & $\begin{array}{l}\text { Odds } \\
\text { Ratio }\end{array}$ & $\begin{array}{ll}95 \% & \text { Confidence } \\
\text { interval } & \end{array}$ & Wald statistic & $\begin{array}{l}\text { Degrees of } \\
\text { freedom }\end{array}$ & $\mathbf{P}$ \\
\hline Age ( $\geq 45$ years $)$ & 3.46 & $2.07-5.78$ & 22.52 & 1 & $<0.001 *$ \\
\hline Hypertension & 5.67 & $3.28-9.82$ & 38.46 & 1 & $<0.001 *$ \\
\hline Abdominal obesity & 31.02 & $15.79-60.92$ & 99.47 & 1 & $<0.001 *$ \\
\hline Hyperglycaemia & 3.78 & $1.86-7.71$ & 13.43 & 1 & $<0.001 *$ \\
\hline Impaired fasting glucose & 3.83 & $1.17-12.53$ & 4.91 & 1 & $0.027 *$ \\
\hline Hypertriglyceridaemia & 3.38 & $1.14-10.02$ & 4.82 & 1 & $0.028^{*}$ \\
\hline Low HDL & 1.98 & $1.14-3.45$ & 5.83 & 1 & $0.016^{*}$ \\
\hline Hypercholesterolaemia & 1.90 & $1.02-3.52$ & 4.09 & 1 & $0.043^{*}$ \\
\hline Metabolic syndrome & 8.33 & $3.68-18.89$ & 25.79 & 1 & $<0.001 *$ \\
\hline Type 2 diabetes & 1.71 & $0.56-5.24$ & 0.88 & 1 & 0.347 \\
\hline High LDL & 1.45 & $0.86-2.42$ & 1.97 & 1 & 0.160 \\
\hline
\end{tabular}

*Statistically significant $\mathrm{P} \leq 0.05$

Table 4: Prevalence of cardiometabolic risk factors of obese subjects and controls according to BMI classes

\begin{tabular}{|c|c|c|c|c|c|c|c|}
\hline \multirow{4}{*}{ RISK FACTOR } & \multicolumn{3}{|c|}{ CONTROLS (\%) } & \multicolumn{4}{|c|}{ OBESE SUBJECTS (\%) } \\
\hline & BMI $\left(\mathrm{Kg} / \mathrm{m}^{2}\right)$ & BMI $\left(\mathrm{Kg} / \mathrm{m}^{2}\right)$ & $\mathrm{P}$ & BMI $\left(\mathrm{Kg} / \mathrm{m}^{2}\right)$ & BMI $\left(\mathrm{Kg} / \mathrm{m}^{2}\right)$ & $\operatorname{BMI}\left(\mathrm{Kg} / \mathrm{m}^{2}\right)$ & $\mathbf{P}$ \\
\hline & $<25.0$ & $25.0-29.9$ & & 30.0-34.9 & $35.0-39.9$ & $>40.0$ & \\
\hline & $\mathrm{n}=104$ & $\mathrm{n}=53$ & & $\mathrm{n}=90$ & $\mathrm{n}=44$ & $\mathrm{n}=16$ & \\
\hline Abdominal obesity & 3.8 & 32.1 & $<0.001 *$ & 72.7 & 96.9 & 100 & $0.001 *$ \\
\hline Hypertension & 15.4 & 26.4 & 0.102 & 47.0 & 71.9 & 100 & $0.025^{*}$ \\
\hline Hypertriglyceridaemia & 2.9 & 3.8 & 0.767 & 9.1 & 9.4 & 16.7 & 0.748 \\
\hline Hypercholesterolaemia & 13.5 & 17.0 & 0.559 & 19.7 & 34.4 & 25.0 & 0.297 \\
\hline High LDL & 26.0 & 37.7 & 0.131 & 37.9 & 43.8 & 25.0 & 0.508 \\
\hline Low HDL & 17.3 & 22.6 & 0.445 & 25.8 & 43.8 & 50.0 & 0.096 \\
\hline IFG & 1.9 & 3.8 & 0.701 & 4.5 & 15.6 & 16.7 & 0.131 \\
\hline Type 2 diabetes & 1.9 & 7.5 & 0.093 & 4.5 & 9.4 & 8.3 & 0.636 \\
\hline Metabolic syndrome & 2.9 & 9.8 & 0.088 & 15.2 & 50.0 & 66.7 & $\begin{array}{l}<0.001 \\
*\end{array}$ \\
\hline
\end{tabular}

*Statistically significant $\mathrm{P} \leq 0.05 ; \mathrm{BMI}$ - body mass index; IFG - impaired fasting glucose

\section{References}

[1]. Misra A, Khurana L. Obesity and the Metabolic Syndrome in developing countries. J Clin Metab, 93(11), 2008, S9-S30.

[2]. Yumuk VD, Hatemi H, Tarakci T, Uyar N, Turan N, Bagriacik N, Ipbuker A. High prevalence of obesity and diabetes mellitus in Konya, a central Anatolian city in Turkey. Diabetes Res Clin Pract, 70, 2005, 151-158.

[3]. Iloh GP, Ikwudinma AO, Obiegbu NP. Obesity and its cardio-metabolic co-morbidities among adult Nigerians in a Primary Care Clinic of a Tertiary Hospital in South-Eastern Nigeria. J Fam Med Primary Care, 2(1), 2013, 20-26.

[4]. Ibarra J. Obesity and cardiovascular disease. The Journal of Lancaster General Hospital, 4(4), 2009, 130-133.

[5]. Qatanani M, Lazar MA. Mechanisms of obesity-associated insulin resistance: many choices on the menu. Genes Dev, 21(1), 2007, 14431455.

[6]. Hall JE, da Silva AA, Brandon E, Stec DE, Ying Z, Jones DW. Pathophysiology of obesity-induced hypertension and target organ damage, in Lip G.P. and Hall J.E. (Eds.), Comprehensive Hypertension, (Elsevier, New York, N.Y., 2007) 447-468.

[7]. Schunkert H. Obesity and target organ damage: the heart. Int J Obes, 26(4), 2002, S15-S20.

[8]. Behan KJ, Mbizo J. The relationship between waist circumference and biomarkers for diabetes and CVD in healthy non-obese women. The Pensacola study. Labmedicine, 38(7), 2007, 422-427.

[9]. Meigs JB, Wilson PF, Fox CS, Vasan RS, Nathan DM, Sullivan LM, D'Agostino RB. Body mass index, metabolic syndrome and risk of type 2 diabetes or cardiovascular disease. J Clin Endocrinol Metab, 91(8), 2006, 2906-2912.

[10]. Friedewald WT, Levy RI, Fredrickson DS. Estimation of the concentration of low-density lipoprotein cholesterol in plasma without the use of preparative ultracentrifugation. Clin Chem. 1972; 18: 499-502.

[11]. Odum EP, Orluwene CG. Metabolic syndrome prevalence in healthy individuals in university of Port Harcourt teaching hospital (UPTH), Port Harcourt. IOSR Journal of Dental and Medical Sciences, 10(3), 2013, 17-22.

[12]. Steinberger J, Daniels SR. Obesity, insulin resistance, diabetes and cardiovascular risk in children: An American Heart Association Scientific Statement from the Atherosclerosis, Hypertension, and Obesity in the Young Committee (Council on Cardiovascular Disease in The Young) and the Diabetes Committee (Council on Nutrition, Physical Activity, and Metabolism). Circulation, 107, 2003, 1448-1453.

[13]. Termizy HM, Mafauzy M. Metabolic syndrome and its characteristics among obese patients attending an obesity clinic. Singapore Med J, 50(4), 2009, 390-394.

[14]. Tataranni PA. Pathophysiology of obesity-induced insulin resistance and type 2 diabetes mellitus. Eur Rev Med Pharmacol Sci, 6, 2002, 2732.

[15]. Siminialayi IM, Emem-Chioma PC, Dapper DV. The prevalence of obesity as indicated by BMI and waist circumference among Nigerian adults attending family medicine clinics as outpatients in Rivers State. Niger J Med, 17(3), 2008, 340-345.

[16]. Mathew B, Francis L, Kayalar A, Cone J. Obesity: Effects on Cardiovascular Disease and its Diagnosis. J Am Board Fam Med, 21(6), 2008, 562-8.

[17]. Hossain P, Kawar B, Nahas ME. Obesity and Diabetes in the Developing World - A Growing Challenge. N Engl J Med, 356, 2007, 213215. 